\title{
El proyecto Peak y sus implicaciones para el acceso a los artículos científicos
}

\begin{abstract}
DURANTE LOS ÚLTIMOS CINCO AÑOS hemos visto cómo las bibliotecas universitarias $y$ también las de investigación han aumentado el número de revistas electrónicas en su fondo bibliográfico, ya sea como complemento a las impresas o bien para reemplazarlas. Por su naturaleza, las publicaciones electrónicas representan retos para los sistemas existentes: tanto las editoriales como las bibliotecas se ven condicionadas por los procedimientos y procesos que reinaban en el entorno del papel impreso. Por ejemplo, las primeras siguen edi-
\end{abstract}

\section{Por Alice Keefer}

tando títulos según el patrón tradicional de recopilaciones de artículos, y las bibliotecas se esfuerzan para adaptar sus presupuestos y operaciones para la integración de suscripciones electrónicas.

Con esta afirmación nuestra intención no es criticar el formato tradicional. Por el contrario, la revista impresa, tal como la conocemos (colecciones de artículos en ejemplares publicados sucesivamente), ha servido con gran éxito para la distribución y difusión de la información científica durante casi 350 años. De todas formas, su gran versatilidad en papel no garantiza necesariamente una adaptación sin problemas al acceso digital.

A medida que se acumula experiencia en la integración de las revistas electrónicas dentro de los fondos bibliográficos, las partes implicadas (sobre todo editoriales y bibliotecas) estudian el uso real de estos recursos a través de informes y estadísticas de transacciones, herramientas que eran inexistentes para medir el uso de revistas impresas.

Un proyecto interesante concluido recientemente estuvo dedi- 
cado al estudio del uso de artículos científicos para explorar nuevas formas de empaquetar la información (bundling) junto con estructuras de tarifas para esta información. El proyecto se llamaba Peak (Pricing electronic access to knowledge, es decir Tarificación del acceso electrónico al conocimiento). Sus principales participantes, la University of Michigan y la editorial Elsevier Science, también colaboraron juntas en un plan pionero sobre revistas-e, Tulip (ver IWE $n$. 42, marzo 96, p. 24-25).

Durante el proyecto la University of Michigan cargó el texto completo de las aproximadamente 1.200 revistas de la editorial mencionada en formato de mapa de bits y también ficheros de texto generados mediante OCR (reconocimiento óptico de caracteres). Entre los 1.200 títulos se incluyeron los de Pergamon, North Holland y Butterworths, todas ellas editoriales previamente adquiridas por Elsevier Science. El servidor llegó a contener 850.000 artículos a los que tuvieron acceso 340.000 usuarios autorizados de 12 instituciones (universidades y organismos de investigación).

El proceso de selección y adquisición de revistas siempre ha supuesto para el biblio-
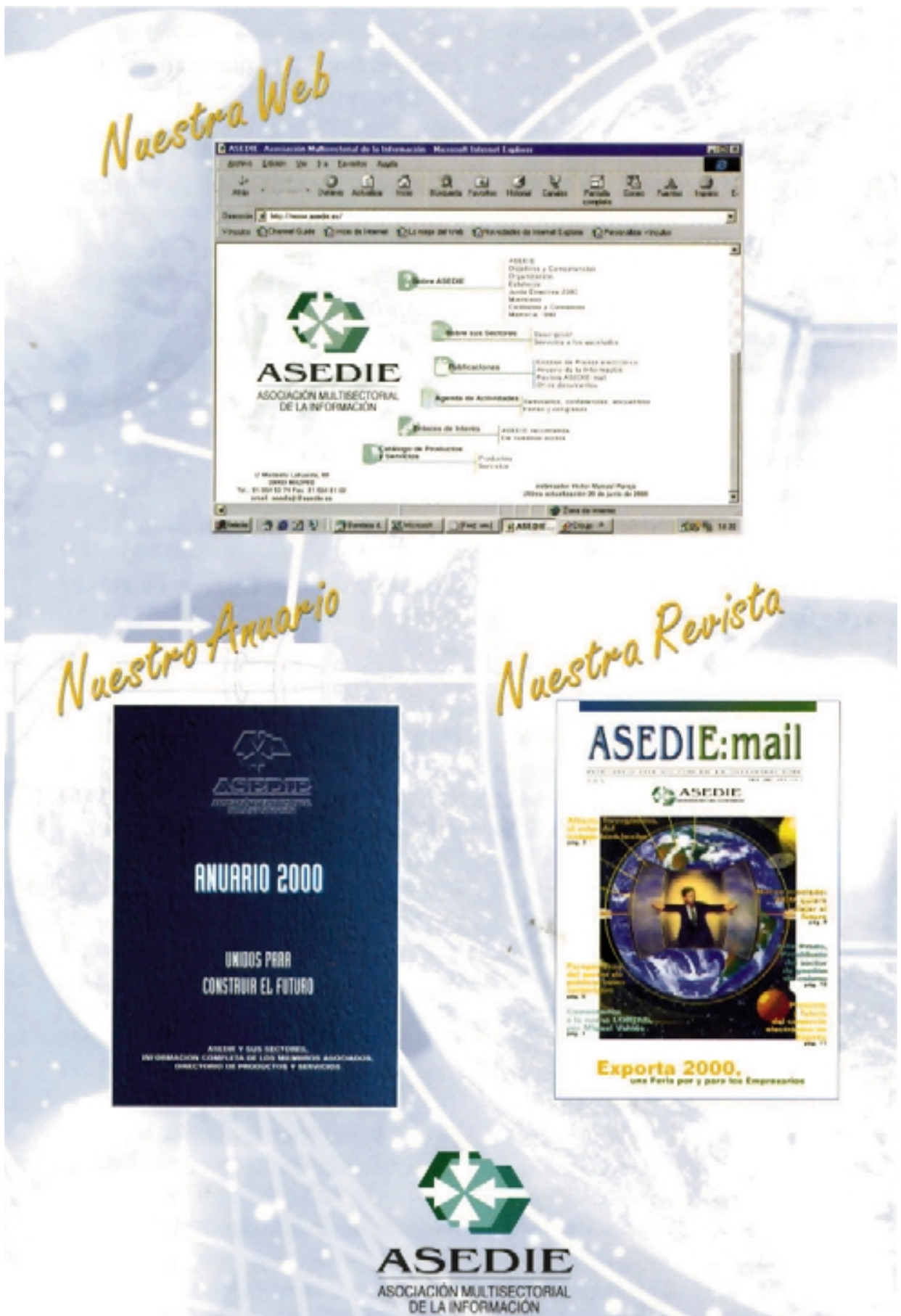

Sede Social en: $c /$ Modesto Lafuente, 60 - 28003 Madrid

Tel.: $915545374 \cdot$ Fax: $915348102 \cdot$ e-mail; asediejt 6 asedie.es * http//www asedie 0

\section{Próximos números monográficos}

Marzo 200I

Mayo 200I

Septiembre 200I
Hosts y productores de bases de datos Información para la inteligencia competitiva Archivos digitales en empresas y organizaciones

Los interesados pueden remitir notas, artículos, propuestas, publicidad, comentarios, etc.,

sobre estos temas a:

epi@sarenet.es 
tecario la toma de una serie de decisiones, pero ello resulta más complejo para la selección de revistas electrónicas: qué títulos adquirir, cuántos ejemplares, mediante subscripciones normales o en forma de bases de datos de texto completo, títulos volcados en servidores locales o accesibles a través de servicios online remotos, versión electrónica solamente o la combinación de recursos electrónicos e impresos... También el bibliotecario se ve obligado a hacer un juicio sobre el futuro valor de la información aún sin publicar, ya que las bibliotecas han de comprometerse a comprar la revista antes de conocer su contenido (es decir, antes de la publicación de los ejemplares, a diferencia de la compra de una monografía cuya petición frecuentemente se gestiona después de que se publique el libro). No obstante, el valor real de la información no se conocerá hasta que haya sido seleccionada y utilizada por el usuario final.

\section{«Al final del segundo año (1999), el 50\% de los artículos adquiri- dos provenían de re- vistas no suscritas por las bibliotecas, resaltando la dificul- tad de prever el valor real de los artículos aún por publicar»}

Para contrarrestar las deficiencias del sistema actual los participantes del proyecto Peak eligieron entre 3 posibles modelos de acceso-e a la información:

- Suscripción tradicional: una vez suscrita la revista, los usuarios autorizados de la institución tenían acceso ilimitado a los artículos.

- Compra individual de artículos (a 7 US\$ cada uno) a petición de los usuarios: cada artículo sólo está disponible con derecho de ac- ceso ilimitado para quien lo adquiere; si otro usuario también quiere tenerlo hay que volver a comprar el artículo pagando la misma tarifa. Este sistema replica los servicios tradicionales de préstamo interbibliotecario u obtención de documentos, con la ventaja de la entrega inmediata de la información solicitada.

- Suscripciones "generalizadas": se pagan por adelantado como las tradicionales y mediante la compra de "vales", pero las "revistas" son virtuales y se configuran a medida que los usuarios van eligiendo artículos para su propio uso. De esta forma el contenido suscrito está determinado posteriormente por la publicación de los artículos y basado en la demanda de los usuarios en vez de la selección preliminar realizada por parte de la editorial.

A diferencia del método anterior (el de la compra de artículos) esta opción permite el posterior uso ilimitado por parte de toda la institución de los artículos adquiridos por un solo usuario. Se considera completa una "suscripción" después de la adquisición de 120 artículos de revistas no suscritas. Este número se basa en el número medio (120) de artículos aparecidos anualmente en una publicación científica. Además de la posibilidad de poder hacer un uso más extenso de los artículos adquiridos en estas revistas post-fabricadas, otra ventaja es que el precio del "vale" es menor que el de la obtención de un artículo por el segundo método. La reducción de coste es posible gracias al compromiso previo (la compra de "vales") por parte de las bibliotecas. Este pago anticipado reduce el riesgo económico para la editorial, de la misma forma que el pago previo del precio de la suscripción tradicional contribuye a la financiación de la publicación de futuros ejemplares. Además, este sistema también satisface la nece- sidad de la biblioteca de trabajar con presupuestos fijos, en vez de enfrentarse con las fluctuaciones presupuestarias relacionadas con los servicios que facturan por transacción.

Unos resultados llamativos de la experiencia Peak son:

- Al final del segundo año (1999) el 50\% de los artículos adquiridos provenían de revistas no suscritas por las bibliotecas, resaltando la dificultad de prever el valor real de artículos aún por publicar.

\section{«Menos del 20\% de los artículos del total de $\mathbf{8 5 0 . 0 0 0}$ disponi- bles habían sido se- leccionados por los usuarios en el tiempo en que duró el pro- yecto. Es decir, más del $\mathbf{8 0} \%$ de los publi- cados no se utilizaron durante este período»}

- Menos del 20\% de los artículos del total de 850.000 disponibles habían sido seleccionados por los usuarios durante el período del proyecto. Es decir, más del $80 \%$ de los publicados no se utilizaron mientras duró la prueba.

Otro asunto destacable fue el impacto del coste sobre el uso de la información. Es conocido el hecho de que la utilización desciende en relación con la aplicación de tarifas. Pero además de éstas también se consideran aspectos de gasto otros conceptos como el tiempo y la comodidad en el uso de la información, los cuales pueden afectar su uso y su valor para el usuario. Factores de costes no económicos identificados por el proyecto Peak son:

1. El número de pantallas que hay que visualizar antes de llegar a la información deseada.

2. Los detalles que el usuario ha de recordar: números de autori- 
zación, contraseñas, datos de tarjetas de crédito, etc.

3. Pasos administrativos para que se reembolsen los gastos de las peticiones.

Tal y como se había previsto, el uso fue menor en aquellos casos en que se interpusieron dificultades para los usuarios.

Las implicaciones inmediatas de los resultados de Peak podrán orientar a las bibliotecas en la selección de opciones para el acceso y la entrega de contenido electrónico. No obstante parece evidente que el conocimiento del comportamiento de los usuarios frente a la información digital también afectará la forma de actuar de las editoriales en cuanto al tipo y formato de información suministrada, las cuales ya han empezado a ofrecer nuevas formas de "empaquetar" la información y nuevas estructuras de precios para satisfacer las necesidades de los usuarios, al tiempo que aprovechan el potencial de los documentos digitales. Seguramente ciertas cifras, como por ejemplo la del porcentaje de artículos publicados que no son utilizados -el $80 \%$ - no pasarán inadvertidas para las editoriales, sobre todo si los nuevos modelos de adquisición se basan más en la compra individual de artículos que en la suscripción de recopilaciones predeterminadas de ellos.

Página de Peak:

http://www.lib.umich.edu/libhome/peak/ overview.html

Gazzale, Robert S.; MacKie-Mason, Jeffrey K. System design, user cost and electronic usa- ge of journals. 2000, 22 de marzo. Consultado en: 30-12-2000.

http://www.si.umich.edu/PEAK-2000/

gazzale.pdf

MacKie-Mason, Jeffrey K.; Riveros, Juan F. "Bundling y el acceso electrónico a la información académica: el proyecto Peak". En: II Seminario internacional complutense de telecomunicaciones e información: retos jurídicos de la información en internet, 1998. Consultado en: 3012-2000.

http://www.lib.umich.edu/libhome/peak/

Complutense.pdf

MacKie-Mason, Jeffrey K.; Riveros, Juan F.; Gazzale, Robert S. "Pricing and bundling electronic information goods: experimental evidence". En: The internet upheaval: raising questions, seeking answers in communications policy. Cambridge, MA: MIT Press, 2000. Consultado en: 30-12-2000.

http://www-personal.umich.edu/ jmm/papers/ peak-tprc99.pdf

Alice Keefer, profesora asociada, Universitat de Barcelona, Facultat de Biblioteconomia i Documentació. akeefer@arrakis.es 